\title{
Induction of apoptosis by VB1 in breast cancer cells: The role of reactive oxygen species and $\mathrm{Bcl}-2$ family proteins
}

\author{
LI-HUA LIU ${ }^{1}$, YING-JUN ZHOU ${ }^{2}$, LAN DING $^{1}$, SHUN-ZHI ZHANG $^{1}$, JI SUN $^{1}$ and JIAN-GUO CAO ${ }^{3}$ \\ ${ }^{1}$ The First Hospital of Changsha, Changsha, Hunan 410005; ${ }^{2}$ School of Pharmacy, Central South University; \\ ${ }^{3}$ Medical College, Hunan Normal University, Changsha, Hunan 410013, P.R. China
}

Received August 16, 2013; Accepted November 18, 2013

DOI: $10.3892 /$ ijmm.2013.1567

\begin{abstract}
We have previously reported that the EVn-50 mixture of vitexins (lignan compounds) containing the purified vitexin (neolignan) compound, 6-hydroxy-4(4-hydroxy-3methoxyphenyl)-3-hydroxymethyl-7-methoxy-3,4-dihydro-2naphthaldehyde, termed VB1, exhibits potent anticancer activity through the induction of apoptosis in several types of cancer cells, including MDA-MB-231 cells. However, the exact molecular mechanisms by which VB1 induces apoptosis in MDA-MB-231 cells have not yet been fully elucidated. In this study, to our knowledge, we provide for the first time mechanistic evidence that VB1-induced apoptosis in the human breast cancer line, MDA-MB-231, is associated with the generation of reactive oxygen species (ROS), the activation of caspases and the modulation of the expression of myeloid leukemia cell differentiation protein 1 (Mcl-1), B cell lymphoma-2 (Bcl-2) and Bcl-2-associated X (Bax) proteins. The silencing of Mcl-1 by RNA interference enhanced VB1-induced apoptosis. In addition, VB1 did not induce ROS generation or apoptosis in the immortalized non-cancerous breast cell line, MCF-10A. Our findings reveal a novel mechanism underlying VB1-induced apoptosis, and highlight VB1 as a promising candidate for the therapy of human breast cancer.
\end{abstract}

\section{Introduction}

Lignans are a group of complex polyphenolic antioxidants found in plants. Clinical studies have suggested that lignans are one of the most promising classes of dietary agents with regard to cancer prevention $(1,2)$. In particular, lignans may prevent hormone-dependent diseases, such as breast and prostate cancer. Several lignans have been extensively investigated in pre-clinical tumor xenograft models, prospective

Correspondence to: Professor Jian-Guo Cao, Medical College, Hunan Normal University, 371 Tongzipo Road, Changsha, Hunan 410013, P.R. China

E-mail: caojianguo2005@126.com

Key words: breast cancer, VB1, apoptosis, reactive oxygen species, Bcl-2 and case-control epidemiological studies, as well as in some clinical trials (2-4). Although no randomized clinical trial data exist to date that support that lignans can reduce cancer growth, there are several biomarker-based neoadjuvant trials, indicating that the dietary intake of flaxseed lignan can inhibit tumor cell proliferation and induce apoptosis (2,5). A previous prospective cohort study indicated that a higher dietary intake of lignan is associated with a reduced risk of hormone-dependent breast cancer (6). We previously reported the isolation of a unique class of lignan compounds termed vitexins, from the seed of the Chinese herb, Vitex negundo (7). Our previous study revealed that the purified vitexin compound, VB1 (neolignan), exerts potent cytotoxic effects and induces apoptosis by decreasing the B cell lymphoma-2 (Bcl-2)/ Bcl-2associated $\mathrm{X}$ (Bax) protein ratio in a number of cancer cell lines (7). However, the molecular mechanisms responsible for the induced apoptosis by VB1 in the human breast cancer cell line, MDA-MB-231, were not fully elucidated.

Dysfunctions in apoptosis can result in the development of cancer and in resistance to anticancer therapeutic agents. Therefore, apoptosis is a mechanism that needs to be exploited when developing novel chemotherapeutic drugs for cancer. The mammary gland, similar to numerous other tissues, expresses a number of proteins of the Bcl-2 family, including Bcl-2, Bax, Bcl-2 homologous antagonist killer (Bak) and Bcl-2-associated death promoter (Bad). Understanding the role that $\mathrm{Bcl}-2$ family members play in regulating the survival of mammary epithelial cells is salient to both normal mammary gland physiology and the development of novel therapeutic approaches for breast cancer. The induced myeloid leukemia cell differentiation protein $1(\mathrm{Mcl}-1)$ protein may be an additional key determinant in the development of various malignancies similar to other members of the anti-apoptotic Bcl-2 protein family; Mcl-1 is expressed in a wide variety of tissues and neoplastic cells (8-10). Mcl-1 has been described as an early and transient survival factor, tightly regulated at the transcriptional and post-transcriptional levels, which allows a cell to either survive by activating other anti-apoptotic genes or to undergo apoptosis (11).

Although lignans are generally considered as antioxidants, they can also generate reactive oxygen species (ROS), depending on their structure and molecular environment $(12,13)$. ROS play an important role in the intrinsic pathway of apoptosis. In human leukemic cells, the accumulation of intracellular ROS 
led to a decrease in the mitochondrial transmembrane potential $\left(\mathrm{MTP} / \Delta \psi_{\mathrm{m}}\right)$, the release of cytochrome $c$, followed by the activation of the caspase cascade, and ultimately, to apoptosis (14). A number of lignans exert direct and indirect pro-oxidant effects by modulating the mitochondrial pathway $(15,16)$. In addition, Wang et al (16) reported that obtusilactone A (OA) and (-)-sesamin act as both inhibitors of the human mitochondrial Lon protease and as DNA damage agents to activate the DNA damage checkpoints and induce apoptosis in non-small cell lung cancer (NSCLC) cells.

The present study was carried out to examine whether VB1 induces apoptosis in the human breast cancer line, MDA-MB-231. We demonstrate that the VB1-mediated induction of apoptosis involves ROS generation, the downregulation of $\mathrm{Mcl}-1 / \mathrm{Bcl}-2$ and the upregulation of Bax in a dose-dependent manner.

\section{Materials and methods}

Reagents. The lignan compound VB1 (6-hydroxy-4-(4-hydroxy3-methoxyphenyl)-3-hydro-methyl-7-methoxy-3,4-dihydro-2naphthaldehyde) (Fig. 1) was purified from the vitexin mixture EVn-50, extracted from the seeds of Vitex negundo, as previously described (7). RPMI-1640 medium and fetal bovine serum (FBS) were supplied by Gibco-BRL (Grand Island, NY, USA). Trypan blue and propidium iodide (PI) were purchased from Sigma-Aldrich (St. Louis, MO, USA). N-acetylcysteine (NAC) and dimethyl sulfoxide (DMSO) were obtained from SigmaAldrich. Z-DEVD-fmk (caspase-3 specific inhibitor), and Ac-LEHD-CHO (caspase-9 specific inhibitor) were obtained from EMD Millipore Corp. (Billerica, MA, USA). Dichlorodihydrofuorescein diacetate (DCHF-DA) was purchased from Molecular Probes Inc. (Eugene, OR, USA). Mouse anti-human Mcl-1, anti-Bcl-2, anti-Bax and anti- $\beta$-actin were obtained from Santa Cruz Biotechnology, Inc. (Santa Cruz, CA, USA). Horseradish peroxidase-conjugated anti-mouse immunoglobulins were purchased from Cell Signaling Technology (Beverly, MA, USA). The commercial anti-breast cancer agent, adriamycin (ADM), was obtained from Sigma-Aldrich, and was used as a positive control for the induction of apoptosis, whereas $0.1 \%$ DMSO was used as a negative control.

Cell culture. The human breast cell lines, MDA-MB-231 (cancerous) and MCF-10A (immortalized, non-cancerous), were obtained from the China Center for Type Culture Collection (CCTCC, Wuhan, China). The cell lines were cultured in RPMI1640 supplemented with $10 \%$ FBS, $1 \%$ non-essential amino acids, $100 \mathrm{U} / \mathrm{ml}$ penicillin and $0.1 \mathrm{mg} / \mathrm{ml}$ streptomycin, and were incubated in a humidified atmosphere of $5 \% \mathrm{CO}_{2}$ at $37^{\circ} \mathrm{C}$.

Cytotoxicity assay. A cytotoxicity assay based on the release of lactate dehydrogenase ( $\mathrm{LDH}$ ) was conducted using a cytotoxicity detection kit (Roche Diagnostics Ltd., Penzberg, Germany) as previously described (17). Cells were treated for $48 \mathrm{~h}$ with various concentrations of VB1, EVn-50 or ADM. Culture medium from each well was collected and transferred into 96-well flat-bottom plates. LDH activity was determined by adding equal volumes of the reaction mixture to each well and incubating for up to $30 \mathrm{~min}$. The absorbance of the samples was measured at $490 \mathrm{~nm}$ using a microplate reader.

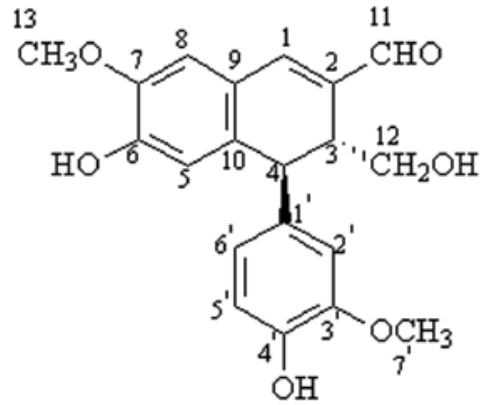

Figure 1. The chemical structure of VB1.

The experiment was repeated three times. Cytotoxicity was calculated using the formula:

Cytotoxicity $(\%)=\frac{\begin{array}{c}\text { Experimental value - } \\ \text { Spontaneous LDH release }\end{array}}{\begin{array}{l}\text { Maximum LDH release - } \\ \text { Spontaneous LDH release }\end{array}} \times 100$

Trypan blue exclusion assay. The cells were treated with various concentrations of VB1 or EVn-50 for $48 \mathrm{~h}$. Trypan blue exclusion assay was carried out as previously descrbibed (18). Three independent experiments were carried out and cell viability was calculated using the following formula:

Cell viability $(\%)=\frac{\text { No. of cells in drug-treated group }}{\text { No. of cells in control group }} \times 100$

Flow cytometry (FCM) with PI staining. Cells were treated with serum-free medium for $24 \mathrm{~h}$ as previously described (19), followed by treatment with medium containing various concentrations of the test agents for $48 \mathrm{~h}$. The cells were then collected and prepared as a single-cell suspension by mechanical blowing with phosphate-buffered saline (PBS), washed with cold PBS twice, fixed with $70 \%$ (v/v) alcohol at $4^{\circ} \mathrm{C}$ for $24 \mathrm{~h}$, and stained with PI. Cell apoptosis was then detected using a flow cytometer (FACS 420; BD Biosciences, Franklin Lakes, NJ, USA).

DNA agarose gel electrophoresis. Cells were treated with serum-free medium for $24 \mathrm{~h}$ as previously described (19), followed by treatment with medium containing various concentrations of the test agents for $48 \mathrm{~h}$. The cells were washed twice with PBS and DNA was extracted using the Apoptotic DNA Ladder detection kit (Bodataike Co., Beijing, China) following the manufacturer's instructions. The extracted DNA was kept at $4^{\circ} \mathrm{C}$ overnight. Subsequently, $8.5 \mu \mathrm{l}$ of DNA were mixed with $1.5 \mu \mathrm{l}$ of $6 \mathrm{X}$ buffer solution, electrophoresed at $40 \mathrm{~V}$ on a $20 \mathrm{~g} / \mathrm{l}$ agarose gel containing ethidium bromide and observed using the DBT-08 gel image analysis system (GBOX EF; Syngene, Cambridge, UK).

Caspase activity assay. MDA-MB-231 cells were plated at a density of $2 \times 10^{4}$ cells/well in Nunc 96 -well plates and cultured overnight under $5 \% \mathrm{CO}_{2}$ at $37^{\circ} \mathrm{C}$. The cells were treated with the test agents for $48 \mathrm{~h}$ to induce apoptosis. Each experiment 
was performed in triplicate. Assays were performed using a Caspase-3 or -9 Activity Assay kit (Beyotime Institute of Biotechnology, Jiangsu, China) following the manufacturer's instructions. Lysates were incubated at $37^{\circ} \mathrm{C}$ for $2 \mathrm{~h}$. Thereafter, the absorbance was measured at $405 \mathrm{~nm}$ in an enzymelabeling instrument (ELX-800; BioTek, Shanghai, China). For the caspase inhibitor assay, cells were pre-treated with the caspase-3-specific inhibitor, Z-DEVD-fmk (20 $\mu \mathrm{mol} / \mathrm{l})$, or the caspase-9-specific inhibitor, Ac-LEHD-CHO $(10 \mu \mathrm{mol} / \mathrm{l})$, for $30 \mathrm{~min}$ prior to the addition of the test agents. Caspase activity was expressed as a percentage of caspase activation relative to the negative control.

Quantification of ROS. Intracellular ROS levels were measured by FCM using the fluorescent probe, DCFH-DA, as previously described (19). Briefly, following treatment with various concentrations of the test agents, the cells were incubated with $10 \mu \mathrm{mol} / 1$ of DCFH-DA for $30 \mathrm{~min}$ at $37^{\circ} \mathrm{C}$ in the dark. The cells were then washed with PBS and analyzed within $30 \mathrm{~min}$ using a flow cytometer equipped with an air-cooled argon laser tuned at $488 \mathrm{~nm}$. The fluorescence signal corresponding to DCFH-DA was collected with a 525-nm band pass filter. As a rule, 10,000 cells were counted for each sample.

RNA interference. siRNAs against Mcl-1 (5'-GAAACGCG GUAAUCGGACUTT-3'), and a non-targeting scrambled siRNA control were synthesized by GenePharma Co., Ltd. (Shanghai, China). Cells were transfected with siRNAs using DharmaFECT-1 (Dharmacon, Lafayette, CO, USA) as outlined by the manufacturer. After $48 \mathrm{~h}$, the transfection medium was replaced with regular medium prior to further analysis. The silencing of protein expression was confirmed by western blot analysis. The distribution of the cells at the sub-G1 cell cycle phase was analyzed using FCM.

Western blot analysis. Cell extracts were prepared in lysis buffer containing $0.14 \mathrm{M} \mathrm{NaCl}, 0.4 \mathrm{M}$ triethanolamine, $0.2 \%$ sodium deoxycholate, $0.5 \% \mathrm{NP}-40,1 \mathrm{mmol} / 1$ phenylmethylsulfonyl fluoride, $4.0 \mu \mathrm{g} / \mathrm{ml}$ aprotinin and $4.0 \mu \mathrm{g} / \mathrm{ml}$ leupeptin. The amount of soluble proteins (Mcl-1/Bcl-2/Bax) was quantified by modified Bradford analysis (Bio-Rad, Hercules, CA, USA). Extracted protein samples ( $15 \mu \mathrm{g} / \mathrm{sample})$ were separated by SDS-PAGE, blotted onto polyvinylidene difluoride membranes (Millipore, Bedford, MA, USA), and probed with anti-Mcl-1, anti-Bcl-2, anti-Bax or anti- $\beta$-actin antibodies. Horseradish peroxidase-conjugated anti-mouse immunoglobulins were used for the secondary reactions. Reactive bands were visualized by chemiluminescence using Tropix ${ }^{\circledR}$ CSPD as the substrate (Invitrogen Life Technologies, Carlsbad, CA, USA). Images were scanned using an AlphaImager 2200 scanner and protein expression levels were quantified by densitometry analysis with AlphaEaseFC software (both from Alpha Innotech, San Leandro, CA, USA).

Statistical analysis. The results are reported as the means \pm standard deviation (SD). Typically, three samples were measured for each experiment and all experiments were repeated at least twice to ensure reproducibility. Statistical differences in the data were assessed using a Student's t-test. A difference was deemed statistically significant at a $\mathrm{P}<0.05$.

\section{Results}

Cytotoxic effect of VBI on MDA-MB-231 cells. Both the vitexin mixture EVn-50 and the purified vitexin, VB1, have been reported to inhibit proliferation and cell death in various types of cancer cells (7). However, to our knowledge, the relative cellular cytotoxicity and the dose effects have not been previously investigated for VB1 in comparison to other compounds. Therefore, we treated the breast cancer cell line, MDA-MB231, with VB1, EVn-50 and the commercial anti-breast cancer agent ADM. Cell death was quantified by an LDH release assay. While treatment with concentrations of VB1 as low as $1.0 \mu \mathrm{mol} / 1$ caused marginal cell death $(\sim 11 \%)$, the compound caused a significant and dose-dependent increase in cell death at higher concentrations (Fig. 2A), with cell death reaching $\sim 83 \%$ upon treatment with $8.0 \mu \mathrm{mol} / 1$ of VB1.

The trypan blue exclusion assay further revealed that VB1 acts as a potent cytotoxic agent in MDA-MB-231 cells, also in a dose-dependent manner. VB1 markedly inhibited the growth of MDA-MB-231 cells, in a similar manner to EVn-50 at the same concentration levels (Fig. 2B). These results suggest that both EVn-50 and VB1 exert intense cytotoxic effects on MDA-MB-231 cells.

Apoptotic effects of VBI on MDA-MB-231 cells. To determine whether VB1 induces apoptosis in human breast cancer cells, the MDA-MB-231 cells were treated with increasing concentrations of VB1 for $48 \mathrm{~h}$. Apoptotic cell death was examined by: i) estimating the cell population at the sub-G1 cell cycle phase using FCM and PI staining to measure corresponding DNA content and ii) observing DNA fragmentation in DNA agarose gels. Fig. 3A illustrates that there is a dose-dependent increase in the percentage of the sub-G1 cell population $(\mathrm{P}<0.05)$, reaching $53.6 \pm 4.2 \%$ after $48 \mathrm{~h}$ of treatment with $8.0 \mu \mathrm{mol} / \mathrm{l} \mathrm{VB} 1$. The potency of VB1 was found to be similar to the mixture of lignan compounds, EVn-50. Furthermore, treatment with $8.0 \mu \mathrm{mol} / \mathrm{l} \mathrm{VB} 1$ for $48 \mathrm{~h}$ resulted in the formation of DNA ladder-like profiles (Fig. 3B). Overall, these results indicate that VB1 effectively induces the apoptotic cell death of MDA-MB-231 cells in a dose-dependent manner.

Caspase activation by VB1 in MDA-MB-231 cells. To determine whether caspases are involved in VB1-induced apoptosis, we first evaluated the activity of caspase- 3 and -9 in the VB1-treated cells. Treatment of the MDA-MB-231 cells with VB1 induced a significant and progressive increase in the levels of active caspase-3 and -9 (Fig. 4). To determine whether VB1-induced apoptosis is mediated by the activation of caspases, we used a caspase-3-specific inhibitor (Z-DEVD-fmk) and a caspase-9-specific inhibitor (Ac-LEHD-CHO). Z-DEVD-fmk and Z-LEHD-fmk prevented the activation of caspase-3 (Fig. 4A) and caspase-9 (Fig. 4B), respectively. Moreover, Fig. 4C illustrates that Z-DEVD-fmk completely abrogated and Ac-LEHD-CHO partly attenuated the VB1-induced accumulation of cells at the sub-G1 phase. These data indicate that VB1-induced apoptosis is essentially dependent on the activation of caspase-3 and -9 in MDA-MB-231 cells.

Effect of VB1 on ROS generation in MDA-MB-231 cells. Since oxidative damage appears to be associated with the anticancer effects of lignans (20), we then examined the levels of intracel- 
A

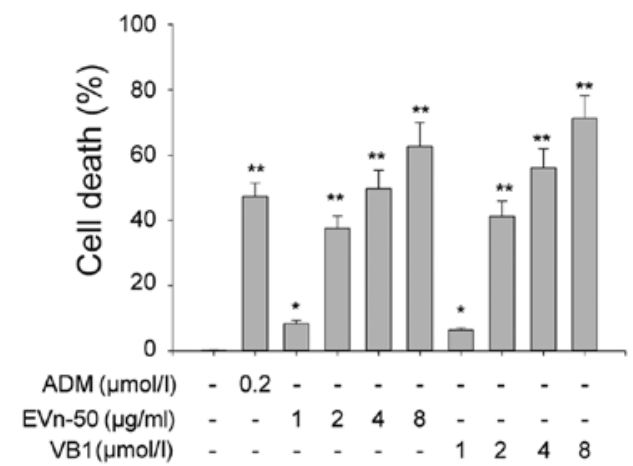

B

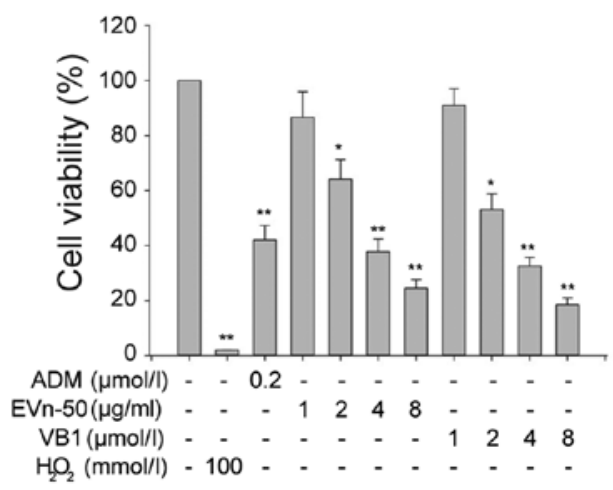

Figure 2. Cytotoxicity of VB1 in MDA-MB-231 cells. (A) Following treatment with various concentrations of VB1, EVn-50 or adriamycin (ADM) for $48 \mathrm{~h}$, cell death percentage was assessed using a cytotoxicity detection kit based on lactate dehydrogenase (LDH) release. (B) Following treatment with various concentrations of VB1, EVn-50 or ADM for $48 \mathrm{~h}$, cell viability was assessed using a trypan blue exclusion assay. The number of viable cells after treatment is expressed as a percentage, relative to the number of viable cells from the vehicle control. Columns represent the mean values from three independent experiments and bars standard deviation (SD). ${ }^{*} \mathrm{P}<0.05$ and ${ }^{* *} \mathrm{P}<0.01$ vs. treatment with the negative control group, where cells were treated with $0.1 \%$ dimethyl sulfoxide (DMSO).

lular ROS in MDA-MB-231 cells following treatment with VB1, using the oxidation-sensitive fluorescent probe, DCFH-DA, which is oxidized to 2',7'-dichlorofluorescein (DCF) in the presence of ROS. As shown in Fig. 5A, treatment of the cells with VB1 increased the mean fluorescence intensity (MFI) of DCF from $35.1 \pm 4.2$ (at $4.0 \mu \mathrm{mol} / \mathrm{l} \mathrm{VB1}$ ) to $72.5 \pm 3.3$ (at $8.0 \mu \mathrm{mol} / \mathrm{l}$ ).

To investigate the potential link between the elevation of intracellular ROS levels and apoptotic cell death in VB1-treated cells, MDA-MB-231 cells were pre-incubated with the antioxidant, NAC (10 mmol/l), prior to treatment with EVn-50 and VB1. VB1 treatment failed to induce ROS generation in the NAC-pre-treated MDA-MB-231 cells. The population of cells at the sub-G1 cell cycle phase was reduced upon treatment with EVn-50 or VB1 in combination with NAC (Fig. 5B). These findings provide evidence that apoptosis induced by VB1 in MDA-MB-231 cells is dependent upon the generation of ROS.

Effects of VB1 on Mcl-1, Bcl-2 and Bax expression in $M D A-M B-231$ cells. The Mcl-1 protein plays an important role in the development of various malignancies and is expressed in a wide variety of tissues and neoplastic cells (7-9). In addi-
A

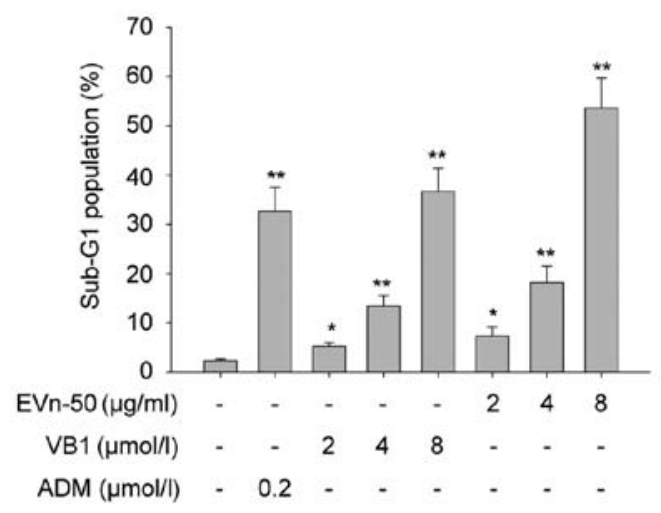

B

$\begin{array}{rllllll}\mathrm{ADM}(\mu \mathrm{mo} / \mathrm{l} /) & 0.0 & 0.1 & 0.0 & 0.0 & 0.0 & 0.0 \\ \mathrm{EVn}-50(\mu \mathrm{g} / \mathrm{ml}) & 0.0 & 0.0 & 4.0 & 8.0 & 0.0 & 0.0 \\ \mathrm{VB}-1(\mu \mathrm{mo} / \mathrm{l} /) & 0.0 & 0.0 & 0.0 & 0.0 & 4.0 & 8.0\end{array}$

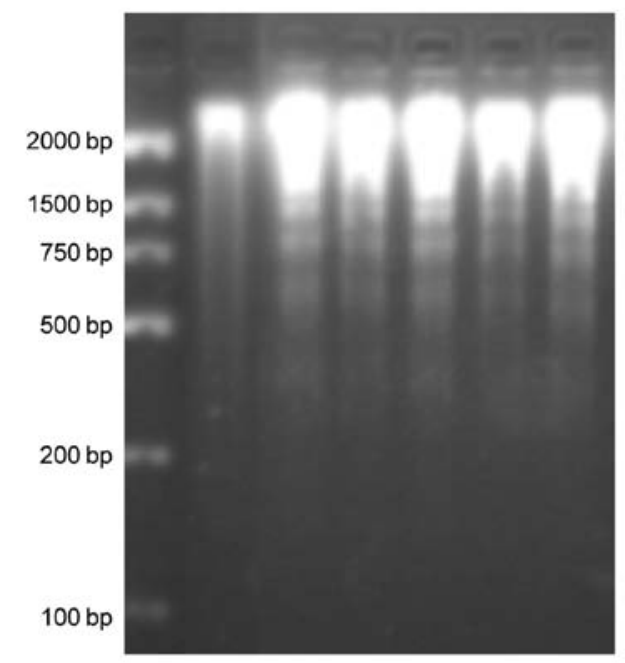

Figure 3. VB1-induced apoptosis in MDA-MB-231 cells. (A) MDA-MB-231 cells were treated with the indicated concentrations of VB1, EVn-50 or adriamycin (ADM) for $48 \mathrm{~h}$ and were subsequently analyzed by flow cytometry. Columns represent the mean values from three independent experiments and bars standard deviations (SD). ${ }^{*} \mathrm{P}<0.05$ and ${ }^{* *} \mathrm{P}<0.01$ vs. treatment with the negative control group, where cells were treated with $0.1 \%$ dimethyl sulfoxide (DMSO). (B) DNA agarose gel showing the formation of ladderlike profiles in the DNA of MDA-MB-231 cells treated with the indicated concentrations of VB1 for $48 \mathrm{~h}$.

tion to its anti-apoptotic effects, Mcl-1 has also been shown to be involved in the regulation of cell cycle progression (21). In addition, Bax and $\mathrm{Bcl}-2$ proteins play crucial roles in apoptosis. Thus, we examined the dose-dependent effects of VB1 on the protein levels of Mcl-1, Bax and Bcl-2 in MDA-MB-231 cells. The expression of the three apoptosis-related proteins was measured by western blot analysis. Treatment of cells with VB1 induced a decrease in Mcl-1 and Bcl-2 expression with a concomitant increase in the protein level of Bax (Fig. 6A). This resulted in a significant increase in the Bax/Bcl-2 and Bax/ Mcl-1 ratios (Fig. 6B and C), which are markers of apoptosis induction.

Mcl-1 downregulation enhances VB1-induced apoptosis in MDA-MB-231 cells. Cellular levels of Mcl-1 have been reported to play an important role in the resistance of tumor 
A

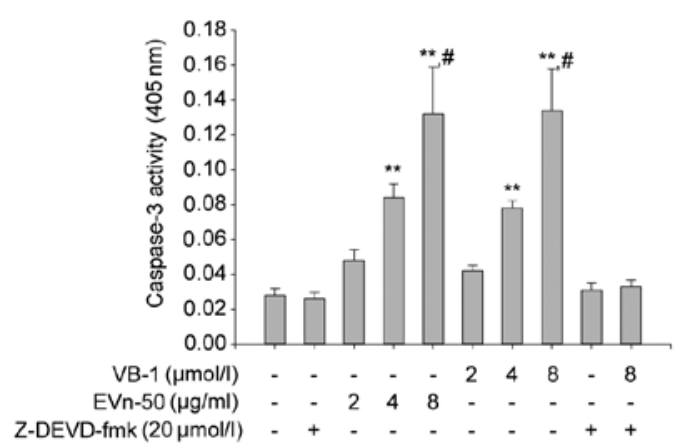

B

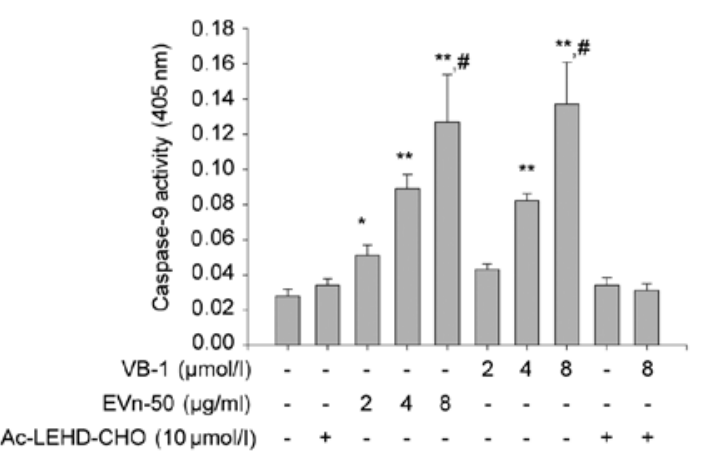

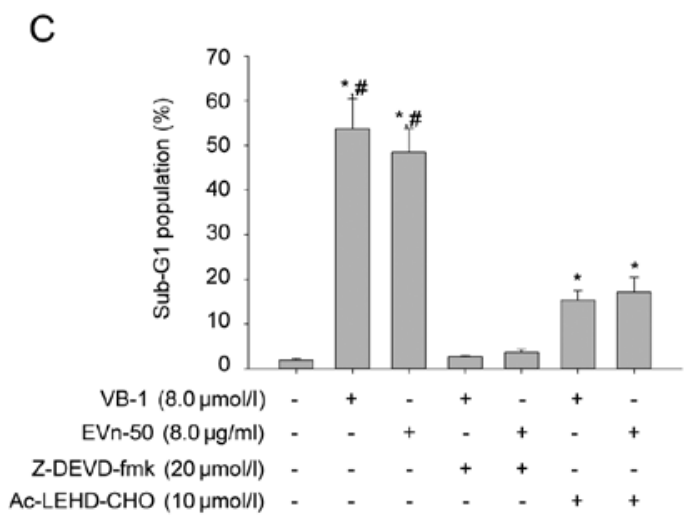

Figure 4. Effects of VB1 on caspase activity and cell cycle distribution in MDA-MB-231 cells. (A) Caspase-3 activity; (B) caspase-9 activity. MDA-MB-231 cells were treated with the indicated concentrations of VB1 for $48 \mathrm{~h}$ and caspase-3 or -9 activities were assayed using corresponding kits. Lysates were incubated at $37^{\circ} \mathrm{C}$ for $2 \mathrm{~h}$. Thereafter, the absorbance was measured at $405 \mathrm{~nm}$ in an enzyme-labeling instrument. In the caspase inhibitor assay, cells were pre-treated with caspase-3 specific inhibitor (Z-DEVD-fmk, $20 \mu \mathrm{mol} / \mathrm{l}$ ) or caspase-9 specific inhibitor (Ac-LEHD-CHO, $10 \mu \mathrm{mol} / \mathrm{l}$ ) for 30 min prior to the addition of the test agents. (C) MDA-MB-231 cells were treated with $8.0 \mu \mathrm{mol} / 1 \mathrm{VB} 1$ or $8.0 \mu \mathrm{g} / \mathrm{ml}$ Evn-50 for $48 \mathrm{~h}$ in the absence or presence of $20 \mu \mathrm{mol} / 1 \mathrm{Z}-\mathrm{DEVD}-\mathrm{fmk}$ or $10 \mu \mathrm{mol} / 1 \mathrm{Ac}-\mathrm{LEHD}-\mathrm{CHO}$. The distribution of cells at the sub-G1 phase was assessed using flow cytometry. Columns represent the mean values from three independent experiments and bars standard deviations $(\mathrm{SD}) .{ }^{*} \mathrm{P}<0.05$ and ${ }^{* * *} \mathrm{P}<0.01$ vs. treatment with the negative control group, where cells were treated with $0.1 \%$ dimethyl sulfoxide (DMSO); ${ }^{*} \mathrm{P}<0.05$ vs. treatment with the Z-DEVD-fmk plus VB1 or Evn-50 and the Ac-LEHD-CHO plus VB1 or Evn-50 groups.

A

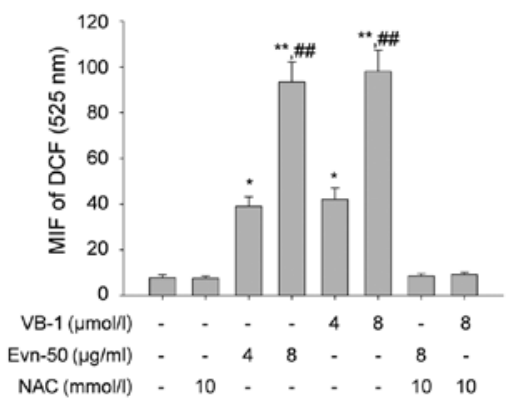

B

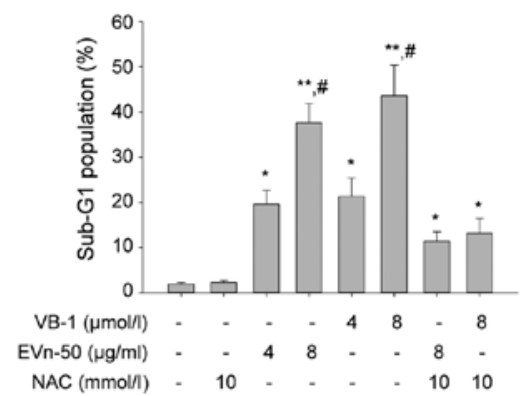

Figure 5. VB1 induces intracellular reactive oxygen species (ROS) accumulation in MDA-MB-231 cells. (A) MDA-MB-231 cells were treated with VB1 (4.0 or $8.0 \mu \mathrm{mol} / \mathrm{l})$ or Evn-50 (4.0 or $8.0 \mu \mathrm{g} / \mathrm{ml})$ for $6 \mathrm{~h}$. A total of $10 \mu \mathrm{mol} / 1$ of dichlorodihydrofuorescein diacetate (DCHF-DA) was added 30 min before collecting cells. The mean fluorescent intensities (MFI) of 10,000 cells were analyzed with a flow cytometer. (B) Distribution of cells at the sub-G1 phase, as assessed by flow cytometry. Columns represent the mean values from three independent experiments and bars standard deviations (SD). DCF, 2',7'-dichlorofluorescein; NAC, $\mathrm{N}$-acetylcysteine. ${ }^{*} \mathrm{P}<0.05$ and ${ }^{* *} \mathrm{P}<0.01$ vs. treatment with the negative control group, where cells were treated with $0.1 \%$ dimethyl sulfoxide (DMSO); ${ }^{*} \mathrm{P}<0.05$ and ${ }^{\# \#} \mathrm{P}<0.01$ vs. treatment with the NAC plus VB1 or Evn-50 group.

cells to chemotherapeutic agents (22). To ascertain whether the downregulation of this anti-apoptotic protein by EVn-50 and VB1 can induce apoptosis, we performed RNA interference experiments with siRNA oligonucleotides specific to Mcl-1 in MDA-MB-231 cells. The level of Mcl-1 was markedly reduced by the specific siRNA (Fig. 7A), whereas a similar concentra- tion of the scrambled siRNA control did not modify Mcl-1 protein expression. More importantly, the silencing of Mcl-1 enhanced the VB1-induced apoptosis of MDA-MB-231 cells (Fig. 7B). These data suggest that the downregulation of the Mcl-1 protein may be involved in the apoptotic effects of VB1 on MDA-MB-231 cells. 

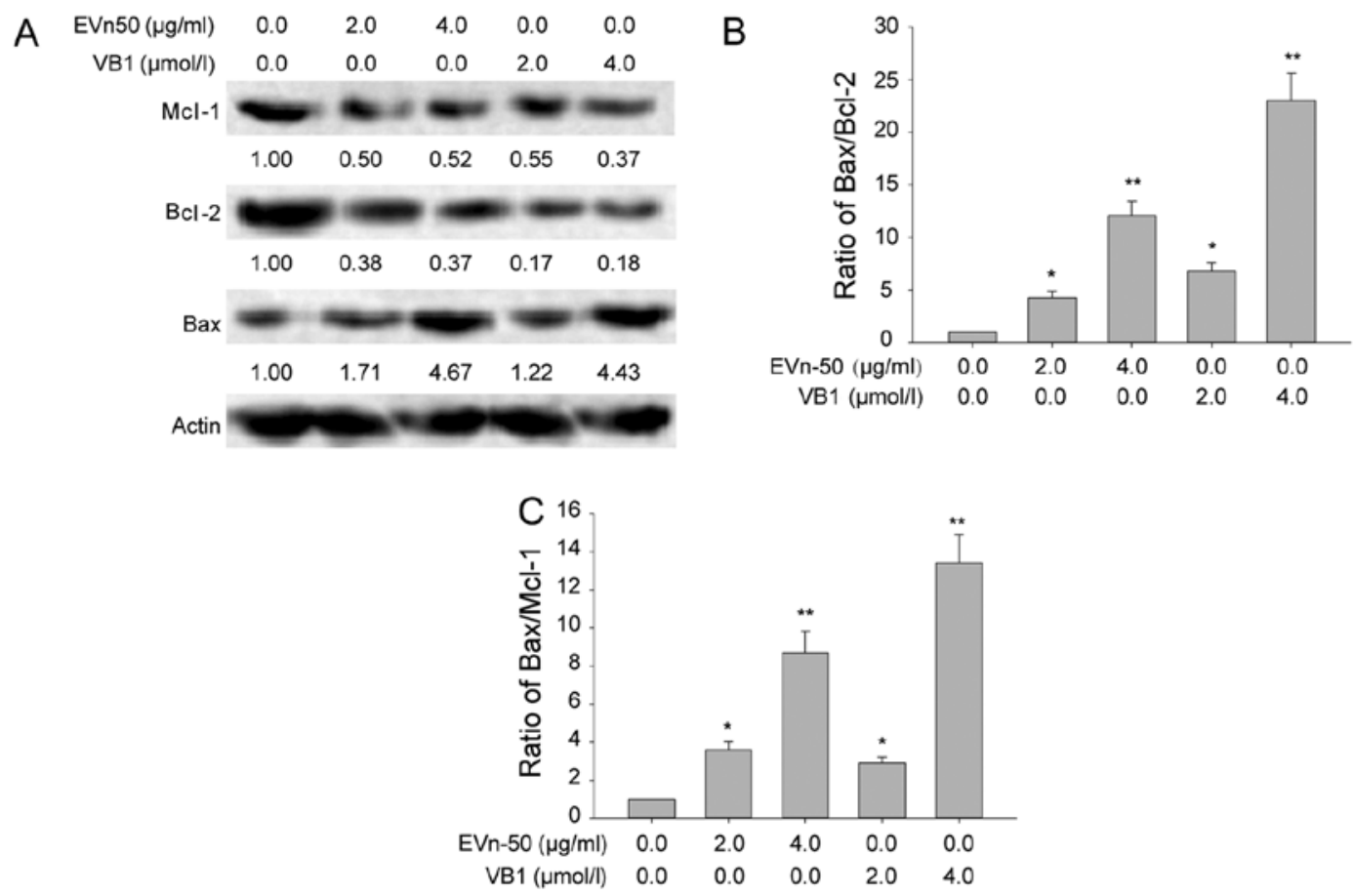

Figure 6.Effect of VB1 on the protein levels of induced myeloid leukemia cell differentiation protein 1 (Mcl-1), B cell lymphoma2 (Bcl-2) and Bcl-2-associated X (Bax) in MDA-MB-231 cells. The cells were treated with VB1 $(2.0$ and $4.0 \mu \mathrm{mol} / \mathrm{l})$, Evn-50 (2.0 and $4.0 \mu \mathrm{g} / \mathrm{ml})$ or dimethyl sulfoxide (DMSO) for $24 \mathrm{~h}$ at $37^{\circ} \mathrm{C}$. (A) Western blot analysis was used to measure the protein levels of Mcl-1, Bcl-2 and Bax, expressed relative to $\beta$-actin as the mean from three independent experiments. We also measured the ratio of (B) Bax/Bcl-2 and (C) Bax/Mcl-1, as a marker of apoptosis induction. VB1 induced an increase in the Bax/Bcl-2 and Bax/ Mcl-1 ratios. ${ }^{*} \mathrm{P}<0.05$ and ${ }^{* *} \mathrm{P}<0.01$ vs. treatment with the negative control group, where cells were treated with $0.1 \%$ dimethyl sulfoxide (DMSO).

A

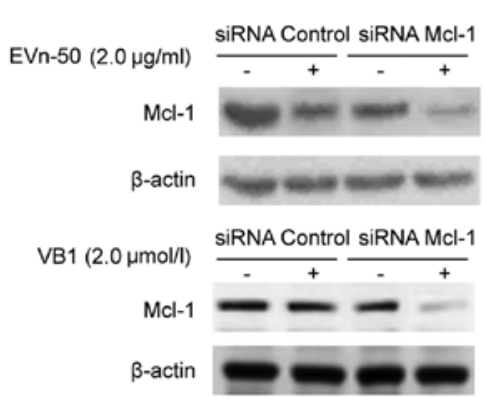

$\mathrm{B}$

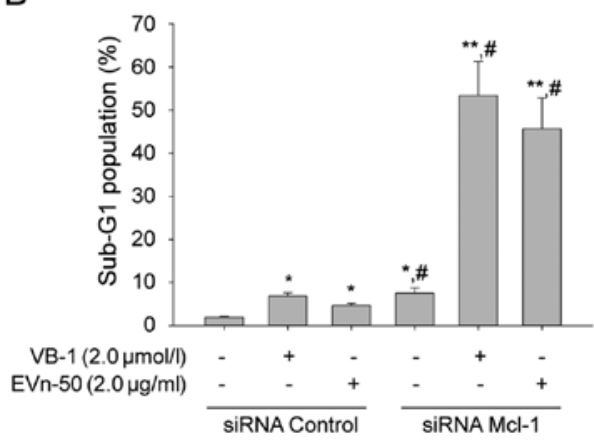

Figure 7. Effect of RNA interference on the protein levels of induced myeloid leukemia cell differentiation protein 1 (Mcl-1) and on VB1-induced apoptosis in MDA-MB-231 cells. (A) The level of Mcl-1 was markedly reduced by the Mcl-1-specific siRNA, whereas a similar concentration of the scrambled siRNA control did not modify Mcl-1 protein expression. (B) Silencing of Mcl-1 enhanced VB1-induced apoptosis. Cells were treated with the test agents for $48 \mathrm{~h}$ in the absence or presence of Mcl-1 siRNA. Their distribution at the sub-G1 phase was assessed using flow cytometry. Columns represent the mean values from three independent experiments and bars standard deviations (SD). $\mathrm{P}<0.05$ and $^{* *} \mathrm{P}<0.01$ vs. treatment with the negative control group, where cells were treated with $0.1 \%$ dimethyl sulfoxide (DMSO); ${ }^{~} \mathrm{P}<0.05$ vs. treatment with the scrambled siRNA control group.
Effects of VBI on apoptosis and ROS generation in MCF-10A cells. Since VB1 induced apoptosis and ROS generation in MDA-MB-231 cells, we then examined the effects of VB1 treatment on the immortalized non-cancerous human breast cell line, MCF-10A. The level of intracellular ROS in MCF-10A cells was not altered in a significant manner upon $3 \mathrm{~h}$ of treatment with any of the tested concentrations of VB1 (Fig. 8A). Furthermore, VB1 did not induce the accumulation of cells at the sub-G1 phase (Fig. 8B). These findings suggest that VB1 specifically induces apoptotic cell death in the breast cancer line, MDA-MB-231.

\section{Discussion}

A number of studies have suggested that lignans may be helpful for cancer prevention and treatment, particularly in the treatment of of breast malignancies $(3,4)$. The lignan compound, VB1, a vitexin purified from EVn-50, belongs to a new class of neolignans. Our previous study demonstrated that VB1, the most abundant vitexin in the EVn-50 mixture, exhibits potent cytotoxic effects on numerous cancer cell lines, regulates the $\mathrm{Bax} / \mathrm{Bcl}-2$ protein ratio and induces apoptosis in MDA-MB-231 cells (7). It has been recently reported that treatment with lignans induces cancer cell apoptosis and is related to the generation of $\operatorname{ROS}(20,23)$. In this study, we firstly demonstrated that VB1 potently induced the cytotoxicity of MDA-MB-231 cells in a dose-dependent manner. The potency of VB1 was similar to that of EVn-50. Secondly, we demonstrated that VB1 induced the apoptosis of MDA-MB-231 cells, accompanied by ROS generation. Finally, we demonstrated that VB1 induced a significant decrease in the expression of 
A

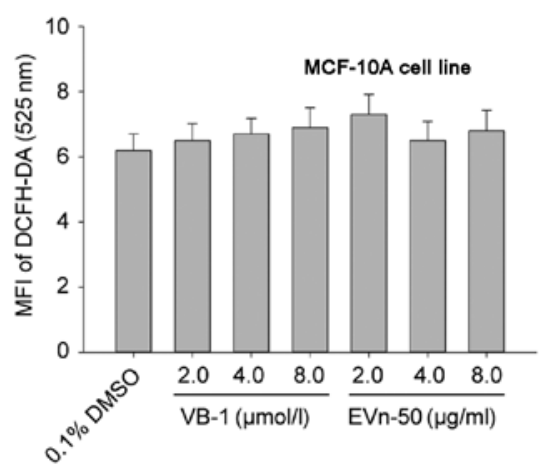

B

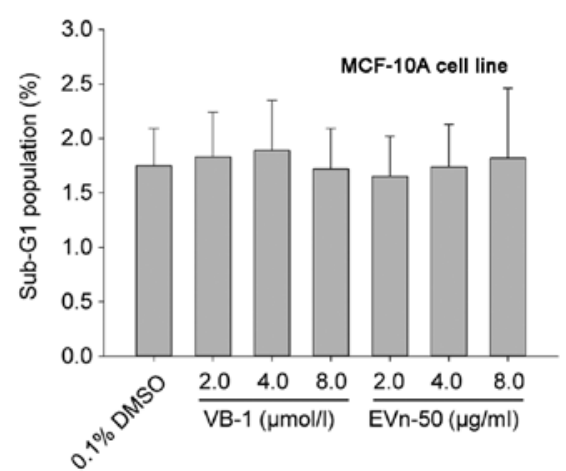

Figure 8. Effects of VB1 on generation of reactive oxygen species (ROS) and on apoptosis in MCF-10A cells. (A) Cells were treated with the indicated concentration of VB1 or EVn-50 for $12 \mathrm{~h}$. A total of $10 \mu \mathrm{mol} / 1$ of dichlorodihydrofuorescein diacetate (DCHF-DA) was added $30 \mathrm{~min}$ before collecting cells. The mean fluorescent intensities (MFI) of 10,000 cells were analyzed with a flow cytometer. (B) Cells were treated with the indicated concentration of VB1 or EVn-50 for $48 \mathrm{~h}$ and the distribution of cells at the sub-G1 phase was analyzed by flow cytometry. Columns represent the mean values from three independent experiments and bars standard deviations (SD).

Mcl-1 and Bcl-2 proteins with a concomitant increase in the protein level of Bax.

ROS have been associated with carcinogenesis but also, paradoxically, with mitochondrial-mediated cell death in cancer cells. The accumulation of ROS is a well-documented central event in mitochondrial-mediated apoptosis (23-25). Intracellular ROS mediate multiple cellular responses, including protein kinase activation (26), cell cycle progression (27), myeloid cell differentiation (28) and apoptotic and necrotic cell death (29). The antioxidant properties of lignans have been associated with their cardioprotective and neuroprotective properties (30), yet no compelling evidence exists to date for a similar association with their preventive properties in cancer. Indeed, the chemopreventive properties of lignans may be due to the elimination of pre-cancerous cells owing to their in vivo pro-oxidant properties. Bose et al (23) argued that the lignan, dihydrobenzofuran, is a potential antitumor agent, inducing $\mathrm{G} 2 / \mathrm{M}$ arrest and apoptosis and involving the generation of ROS. In the present study, we found that another lignan compound, the vitexin VB1, promotes the accumulation of ROS in a concentration-dependent manner in MDA-MB231 cells. NAC is a well-known antioxidant agent, mainly regarded as a ROS scavenger. NAC reduces ROS generation and protects the cells from oxidative stress. It was previously reported that arsenic trioxide-induced ROS generation is inhib- ited by NAC treatment (31). In this study, we showed that the VB1-induced apoptosis of MDA-MB-231 cells is accompanied by ROS generation. We used NAC as an antioxidant to further investigate ROS generation induced by VB1. NAC treatment significantly reduced ROS generation in combination with either EVn-50 or VB1. These results indicate that the induction of ROS may play a crucial role in VB1-induced apoptosis in the MDA-MB-231 cell line.

A number of different members of the Bcl-2 protein family, including Bcl-2, Bax, Bak and Bad are expressed in mammary gland tissue, similar to numerous tissues. Different Bcl-2 family members are commonly overexpressed or underexpressed in human breast cancers. The downregulation of Bcl-2 in human breast cancer cells has been associated with a good prognosis, while decreased Bax expression has been linked to poor clinical outcome. Understanding the role that Bcl-2 family members play in regulating the survival of mammary epithelial cells is salient for both the apprehension of the physiology of healthy mammary gland tissue and the development of novel therapeutic approaches for breast cancer. We demonstrated that the treatment of MDA-MB-231 cells with VB1 induces a marked decrease in Bcl-2 expression, with a concomitant increase in the protein level of Bax, in agreement with a previous study on MDA-MB-231 cells (7). In addition, a certain studies have indicated that Mcl-1, similar to other members of the anti-apoptotic Bcl-2 protein family, may play key roles in the development of various malignancies and is expressed in a wide variety of tissues and neoplastic cells (8-10). The anti-apoptotic activity of Mcl-1 has been clearly established. However to date, only limited information is available on the role that Mcl-1 plays in the biology of solid tumors, and virtually no information is available on its potential role in human solid tumors. Mcl-1 has been described as an early and transient survival factor, tightly regulated at the transcriptional and post-transcriptional levels, that allows a cell to either survive by activating other anti-apoptotic genes or to undergo apoptosis (12). Since the overexpression of anti-apoptotic proteins is an alternative means of inhibiting apoptosis in neoplastic cells $(32,33)$, we investigated the effect of Mcl-1 expression on the human breast cancer cell line, MDA-MB-231, following treatment with VB1. We demonstrated that the downregulation of the Mcl-1 protein induces apoptosis and enhances VB1-induced apoptotic cell death in the MDA-MB-231 cells. Overall in this study, we demonstrate that VB1 is a potent inducer of apoptosis by regulating the levels of proteins related to this process. More importantly, our findings indicate that VB1 specifically induces apoptosis in the human breast cancer cell line, MDA-MB-231, but not in the noncancerous line, MCF-10A, although the mechanisms underlying this specificity remain unknown. Our findings suggest that VB1 is an antitumor agent with specific effects.

In conclusion, the present study demonstrates that VB1 promotes the accumulation of intracellular ROS, resulting in a substantial increase in the Bax/Bcl-2 and Bax/Mcl-1 ratios, leading to apoptosis in the MDA-MB-231 human breast cancer cells. Further investigations are required to provide evidence for the in vivo potency of this potentially therapeutic agent in human breast cancer cells e.g., by using a nude mouse model. Our data highlight a new mechanism underlying VB1-induced apoptosis, and suggest that VB1 may qualify as a highly effective and promising candidate for the therapy of human breast cancer. 


\section{Acknowledgements}

This study was supported by a 2008 grant (no. 2008SK2005) from the Key Project in Science and Technology Pillar Program of Hunan Province.

\section{References}

1. Kim KH, Moon E, Kim SY, Choi SU and Lee KR: Lignan constituents of Tilia amurensis and their biological evaluation on antitumor and anti-inflammatory activities. Food Chem Toxicol 50: 3680-3686, 2012.

2. Demark-Wahnefried W, Price DT, Polascik TJ, Robertson CN, Anderson EE, Paulson DF, Walther PJ, Gannon M and Vollmer RT: Pilot study of dietary fat restriction and flaxseed supplementation in men with prostate cancer before surgery: exploring the effects on hormonal levels, prostate-specific antigen, and histopathologic features. Urology 58: 47-52, 2001.

3. Bergman Jungeström M, Thompson LU and Dabrosin C: Flaxseed and its lignans inhibit estradiol-induced growth, angiogenesis, and secretion of vascular endothelial growth factor in human breast cancer xenografts in vivo. Clin Cancer Res 13: 1061-1067, 2007

4. Zaineddin AK, Buck K, Vrieling A, Heinz J, Flesch-Janys D, Linseisen $\mathrm{J}$ and Chang-Claude $\mathrm{J}$ : The association between dietary lignans, phytoestrogen-rich foods, and fiber intake and postmenopausal breast cancer risk: a German case-control study. Nutr Cancer 64: 652-665, 2012.

5. White Y, Hamada T, Yoshimitsu M, Nakashima M, Hachiman M, Kozako T, Matsushita K, Uozumi K, Suzuki S Kofune H, Furukawa T and Arima N: Novel cytotoxic isolated from Jamaican Hyptis verticillata jacq induces apoptosis and overcomes multidrug resistance. Anticancer Res 31: 4251-4257, 2011.

6. Suzuki R, Rylander-Rudqvist T, Saji S, Bergkvist L, Adlercreutz H and Wolk A: Dietary lignans and postmenopausal breast cancer risk by oestrogen receptor status: a prospective cohort study of Swedish women. Br J Cancer 98: 636-640, 2008.

7. Zhou Y, Liu YE, Cao J, Zeng G, Shen C, Li Y, Zhou M, Chen Y, $\mathrm{Pu}$ W, Potters L and Shi YE: Vitexins, nature-derived lignan compounds, induce apoptosis and suppress tumor growth. Clin Cancer Res 15: 5161-5169, 2009.

8. Glaser SP, Lee EF, Trounson E, Bouillet P, Wei A, Fairlie WD, Izon DJ, Zuber J, Rappaport AR, Herold MJ, Alexander WS, Lowe SW, Robb L and Strasser A: Anti-apoptotic Mcl-1 is essential for the development and sustained growth of acute myeloid leukemia. Genes Dev 26: 120-125, 2012.

9. Krajewski S, Bodrug S, Krajewska M, Shabaik A, Gascoyne R, Berean K and Reed JC: Immunohistochemical analysis of Mcl-1 protein in human tissues. Differential regulation of Mcl-1 and Bcl-2 protein production suggests a unique role for Mcl-1 in control of programmed cell death in vivo. Am J Pathol 146: $1309-1319,1995$.

10. Zhang M, Guo R, Zhai Y and Yang D: LIGHT sensitizes IFNgamma-mediated apoptosis of MDA-MB-231 breast cancer cells leading to down-regulation of anti-apoptosis Bcl-2 family members. Cancer Lett 195: 201-210, 2003.

11. Craig RW: MCL1 provides a window on the role of the BCL2 family in cell proliferation, differentiation and tumorigenesis. Leukemia 16: 444-454, 2002.

12. Bernini R, Gualandi G, Crestini C, Barontini M, Belfiore MC, Willför S, Eklund $\mathrm{P}$ and Saladino R: A novel and efficient synthesis of highly oxidized lignans by a methyltrioxorhenium/hydrogen peroxide catalytic system. Studies on their apoptogenic and antioxidant activity. Bioorg Med Chem 17: 5676-5682, 2009.

13. Apraiz A, Idkowiak-Baldys J, Nieto-Rementería N, Boyano MD, Hannun YA and Asumendi A: Dihydroceramide accumulation and reactive oxygen species are distinct and nonessential events in 4-HPR-mediated leukemia cell death. Biochem Cell Biol 90: 209-223, 2012

14. Jing Y, Dai J, Chalmers-Redman RM, Tatton WG and Waxman S: Arsenic trioxide selectively induces acute promyelocytic leukemia cell apoptosis via a hydrogen peroxide-dependent pathway. Blood 94: 2102-2111, 1999.
15. Lin S, Fujii M and Hou DX: Molecular mechanism of apoptosis induced by schizandrae-derived lignans in human leukemia HL-60 cells. Food Chem Toxicol 46: 590-597, 2008.

16. Wang HM, Cheng KC, Lin CJ, Hsu SW, Fang WC, Hsu TF, Chiu CC, Chang HW, Hsu CH and Lee AY: Obtusilactone A and (-)-sesamin induce apoptosis in human lung cancer cells by inhibiting mitochondrial Lon protease and activating DNA damage checkpoints. Cancer Sci 101: 2612-2620, 2010.

17. Wang X, Ju W, Renouard J, Aden J, Belinsky SA and Lin Y: 17-allylamino-17-demethoxygeldanamycin synergistically potentiates tumor necrosis factor-induced lung cancer cell death by blocking the nuclear factor-kappaB pathway. Cancer Res 66 : 1089-1095, 2006.

18. Zhang YQ, Tang XQ, Sun L, Dong L, Qin Y, Liu HQ, Xia H and Cao JG: Rosiglitazone enhances fluorouracil-induced apoptosis of HT-29 cells by activating peroxisome proliferator-activated receptor gamma. World J Gastroenterol 13: 1534-1540, 2007.

19. Yang XH, Zheng X, Cao JG, Xiang HL, Liu F and Lv Y: 8-Bromo-7-methoxychrysin-induced apoptosis of hepatocellular carcinoma cells involves ROS and JNK. World J Gastroenterol 16: 3385-3393, 2010

20. Fried LE and Arbiser JL: Honokiol, a multifunctional antiangiogenic and antitumor agent. Antioxid Redox Signal 11: 1139-1148, 2009.

21. Salerni BL, Bates DJ, Albershardt TC, Lowrey $\mathrm{CH}$ and Eastman A: Vinblastine induces acute, cell cycle phase-independent apoptosis in some leukemias and lymphomas and can induce acute apoptosis in others when Mcl-1 is suppressed. Mol Cancer Ther 9: 791-802, 2010.

22. Bolesta E, Pfannenstiel LW, Demelash A, Lesniewski ML, Tobin M, Schlanger SE, Nallar SC, Papadimitriou JC, Kalvakolanu DV and Gastman BR: Inhibition of Mcl-1 promotes senescence in cancer cells: implications for preventing tumor growth and chemotherapy resistance. Mol Cell Biol 32: 1879-1892, 2012

23. Bose JS, Gangan V, Prakash R, Jain SK and Manna SK: A dihydrobenzofuran lignan induces cell death by modulating mitochondrial pathway and G2/M cell cycle arrest. J Med Chem 52: 3184-3190, 2009.

24. Lee YJ, Lee DH, Cho CK, Chung HY, Bae S, Jhon GJ, Soh JW, Jeoung DI, Lee SJ and Lee YS: HSP25 inhibits radiation-induced apoptosis through reduction of PKCdelta-mediated ROS production. Oncogene 24: 3715-3725, 2005.

25. Busija DW, Gaspar T, Domoki F, Katakam PV and Bari F: Mitochondrial-mediated suppression of ROS production upon exposure of neurons to lethal stress: mitochondrial targeted preconditioning. Adv Drug Deliv Rev 60: 1471-1477, 2008.

26. Torres M and Forman HJ: Redox signaling and the MAP kinase pathways. Biofactors 17: 287-296, 2003.

27. Boonstra J and Post JA: Molecular events associated with reactive oxygen species and cell cycle progression in mammalian cells. Gene 337: 1-13, 2004.

28. Batista EL Jr, Warbington M, Badwey JA and Van Dyke TE: Differentiation of HL-60 cells to granulocytes involves regulation of select diacylglycerol kinases (DGKs). J Cell Biochem 94: 774-793, 2005.

29. Orrenius S, Gogvadze V and Zhivotovsky B: Mitochondrial oxidative stress: implications for cell death. Annu Rev Pharmacol Toxicol 47: 143-183, 2007.

30. Chen CM, Liu SH and Lin-Shiau SY: Honokiol, a neuroprotectant against mouse cerebral ischaemia, mediated by preserving $\mathrm{Na}^{+}, \mathrm{K}^{+}$-ATPase activity and mitochondrial functions. Basic Clin Pharmacol Toxicol 101: 108-116, 2007.

31. Sánchez Y, Amrán D, Fernández C, de Blas E and Aller P: Genistein selectively potentiates arsenic trioxide-induced apoptosis in human leukemia cells via reactive oxygen species generation and activation of reactive oxygen species-inducible protein kinases (p38-MAPK, AMPK). Int J Cancer 123: $1205-1214,2008$

32. Reed JC: Bcl-2: prevention of apoptosis as a mechanism of drug resistance. Hematol Oncol Clin North Am 9: 451-473, 1995.

33. Strasser A, Huang DC and Vaux DL: The role of the bcl-2/ced-9 gene family in cancer and general implications of defects in cell death control for tumourigenesis and resistance to chemotherapy. Biochim Biophys Acta 1333: F151-F178, 1997. 